\title{
Preparation of S-Glycoside Surfactants and Cysteine Thioglycosides Using Minimally Competent Lewis Acid Catalysis
}

\author{
Lajos Z. Szabó, Dillon J. Hanrahan, Evan M. Jones, Erin Martin, \\ Jeanne E. Pemberton and Robin Polt*
}

Department of Chemistry and Biochemistry

$\mathrm{BIO5}$

The University of Arizona

1306 E. University Blvd.

Tucson, AZ 85721

${ }^{*}$ Corresponding author

polt@u.arizona.edu

(520) 370-2654

25 November 2015 


\title{
Table of Contents/Abstract Graphic.
}

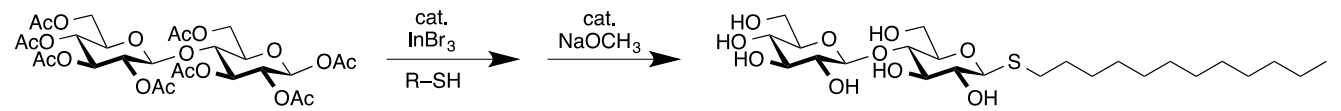

\begin{abstract}
.
Here we report a method for the preparation of anomerically pure $\beta$-S-glycopyranosides (1,2-trans-glycosides) from the corresponding peracetate donors. S-glycosylation was performed in $\mathrm{CHCl}_{3}$ at reflux in the presence of a catalytic amount of $\mathrm{InBr}_{3}$. Deacylation of the intermediate peracetates were achieved under Zemplén conditions. Five pyranose examples, monosaccharides D-glucose and D-galactose and disaccharides cellobiose, maltose, and lactose, were used as donors, and five thiols including an $\alpha / \omega$ dithiol and Fmoc-L-cysteine were used as acceptors. Melting points, high res $M S,[\alpha]_{D}$ and NMR data $\left({ }^{1} \mathrm{H}\right.$ and ${ }^{13} \mathrm{C}$, including COSY, HSQC and $\left.\mathrm{HMBC}\right)$ are reported for compounds not previously described.
\end{abstract}

Keywords: thioglycoside, S-glycosidic linkage, glycolipid, $\operatorname{InBr}_{3}$, surfactant, glycosylated cysteine 
Glycolipids represent a diverse class of compounds typically found in biological membranes ${ }^{1}$ and are regarded as environmentally friendly bio-surfactants with broad use in cosmetics, food, environmental and other industrial applications, ${ }^{2}$ as well as potential therapeutic drug delivery systems. ${ }^{3,4}$ Despite high surface activity, ${ }^{5}$ high biodegradability, and low toxicity, their use is still hampered by a lack of knowledge regarding physical properties, including phase behavior. Even less is known about the S-linked thioglycosides. Thioglycosides (S-glycosides) ${ }^{6}$ may be regarded as relatively inert moieties in vivo, ${ }^{7}$ or as chemical intermediates that can be oxidatively activated ${ }^{8}$ to generate glycoside donors. ${ }^{9-11}$ Thioglycosides were previously prepared from monosaccharide peracetates using a molar excess of $\mathrm{BF}_{3} \cdot \mathrm{Et}_{2} \mathrm{O}$ or $\mathrm{SnCl}_{4}$ as promoters by Kihlberg in the 1990's. ${ }^{12}$ Here we disclose the catalytic use of a much weaker Lewis acid, $\operatorname{lnBr}_{3},{ }^{13}$ which we dub a "minimally competent" Lewis acid. ${ }^{14}$ It should be noted that $\operatorname{InBr}_{3}$ and other Lewis acids have been used in conjunction with the O-glycosylation of trichloroacetimidate donors. ${ }^{15}$ We have previously reported the catalytic use of $\operatorname{lnBr}_{3}{ }^{14}$ and other Lewis acids ${ }^{16}$ to generate O-glycosides from weakly electrophilic peracetate sugars.

The surfactant $\beta$-S-octyl-glucopyranoside and several of its analogues were previously prepared by S-alkylation of 2-(2,3,4,6-tetra-O-acetyl- $\beta$-glucopyranosyl) thiopseudourea $\cdot \mathrm{HBr}$ by Saito and Tsuchiya. ${ }^{17}$ The present approach (Figure 1) seems to be superior, both in terms of the number of steps and in overall yield. $\beta$-DGlucopyranose peracetate 1 was treated with two molar equivalents of 1-octanethiol in $\mathrm{CHCl}_{3}$ at reflux for 1 hour in the presence of $1 \% \operatorname{lnBr}_{3}$, to form the desired $\beta$-peracetate, which was purified by short column chromatography $\left(\mathrm{SiO}_{2}\right)$. No $\alpha$ anomer was 
observed. We propose that the formation of the S-glycoside occurs via a similar route to the previously published O-glycoside example, resulting in only the $\beta$-S-glycoside. ${ }^{14}$ The peracetate product was deprotected using Zemplén deacylation ${ }^{18}$ to provide the desired surfactant. ${ }^{17}$ The glycosylation produced a similar yield in $\mathrm{PhCH}_{3}$, but the reaction in $\mathrm{CH}_{3} \mathrm{CN}$ decreased the yield.

Figure 1. Preparation of $\beta$-Thioglycosides.

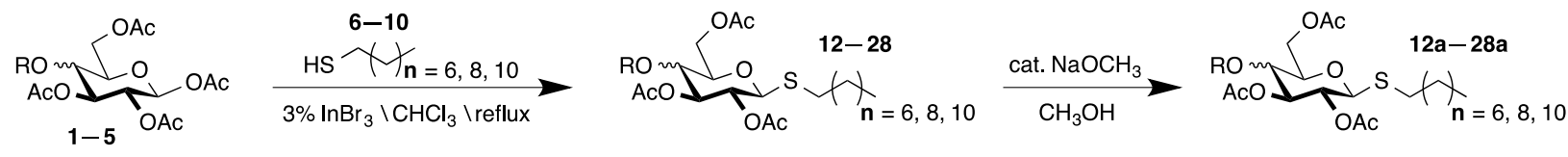

Several mono- and disaccharide peracetates (1-5) and thiols (6-11, Table 1) were subjected to the reaction conditions to provide a variety of S-glycosides. Bolaamphiphile 15a and mono-glycoside $16 \mathbf{a}$ were prepared by adjusting the stoichiometry of the glycosyl donor and acceptor to favor either mono- or di-glycosylation of the $\alpha / \omega$ dithiol. 


\section{Table 1. Results of $\operatorname{InBr}_{3}$ Promoted Glycosylations}

Thioglycosides from $\mathrm{InBr}_{3}$ promoted glycosylations of various thiolipids (6-10) and Fmoc-L-cysteine (11) with five peracetylated mono- and disaccharides (1-5) to produce compounds 12-33. After Zemplén deacylation of the lipids, compounds 12a-28a were isolated and characterized. Compounds $\mathbf{1 2 a},{ }^{19,}{ }^{20} \mathbf{1 3 a},{ }^{20} \mathbf{1 4 a},{ }^{20} \mathbf{1 7 a},{ }^{19,21} \mathbf{1 8 a},{ }^{22} \mathbf{1 9 a},{ }^{22} \mathbf{2 0 a},{ }^{23}$ 22a, ${ }^{24} \mathbf{2 3 a}{ }^{23} \mathbf{2 4 a},{ }^{25} \mathbf{2 5 a},{ }^{24} \mathbf{2 6 a},{ }^{23} \mathbf{2 8 a},{ }^{26} 29,{ }^{27}$ and $\mathbf{3 0}{ }^{28,}{ }^{29}$ have been previously reported using alternate reaction conditions. Previously reported deacylated thioglycosides are noted in Lit. \% indicates combined yields.

\begin{tabular}{|c|c|c|c|c|c|c|c|c|c|c|c|c|c|c|c|c|c|c|c|c|c|c|c|c|}
\hline Donors & \multicolumn{24}{|c|}{ Sulfide Acceptors } \\
\hline & $R^{\prime}=$ & \multicolumn{4}{|c|}{$\begin{array}{c}-\mathrm{S}-\mathrm{C}_{8} \mathrm{H}_{17} \\
6\end{array}$} & \multicolumn{4}{|c|}{$\begin{array}{c}-\mathrm{S}-\mathrm{C}_{10} \mathrm{H}_{21} \\
7\end{array}$} & \multicolumn{4}{|c|}{$\begin{array}{c}\xi-\mathrm{S}-\mathrm{C}_{12} \mathrm{H}_{25} \\
8\end{array}$} & \multicolumn{4}{|c|}{$\begin{array}{c}\xi-\mathrm{S}-\mathrm{C}_{10} \mathrm{H}_{20}-\mathrm{S}-\xi \\
9\end{array}$} & \multicolumn{4}{|c|}{$\begin{array}{c}-\mathrm{S}-\mathrm{C}_{10} \mathrm{H}_{20}-\mathrm{SH} \\
10\end{array}$} & \multicolumn{3}{|c|}{$\mathrm{CO}_{2} \mathrm{H}$} \\
\hline & $\mathrm{R}=$ & $\mathrm{Ac}$ & $\mathrm{H}$ & Lit & $\%$ & $A c$ & $\mathrm{H}$ & Lit & $\%$ & $A c$ & $\mathrm{H}$ & Lit & $\%$ & $A c$ & $\mathrm{H}$ & Lit & $\%$ & $A c$ & $\mathrm{H}$ & Lit & $\%$ & $\mathrm{Ac}$ & Lit & $\%$ \\
\hline Ros & & 12 & $12 a$ & 19,20 & 81 & 13 & $13 a$ & 20 & 88 & 14 & $14 a$ & 20 & 84 & 15 & $15 a$ & - & 86 & 16 & $16 a$ & - & 69 & 29 & 27 & 72 \\
\hline OROR & & 17 & $17 a$ & 19,21 & 89 & 18 & $18 a$ & 22 & 90 & 19 & $19 a$ & 22 & 92 & & & & & & & & & 30 & $\begin{array}{l}28, \\
29\end{array}$ & 81 \\
\hline${ }_{\mathrm{ROR}}^{\mathrm{OR}}$ & $R^{\prime}$ & 20 & $20 \mathrm{a}$ & 23 & 88 & 21 & 21a & - & 66 & 22 & $22 a$ & 24 & 76 & & & & & & & & & 31 & - & 65 \\
\hline $\mathrm{RO}_{\substack{\mathrm{RO} \\
\mathrm{RO}}}^{\mathrm{S}_{4}^{\mathrm{OR}}}$ & & 23 & $23 a$ & 23 & 85 & 24 & $24 a$ & 25 & 87 & 25 & $25 a$ & 24 & 88 & & & & & & & & & 32 & - & 85 \\
\hline$\underbrace{O R O R}_{O R}$ & & 26 & $26 a$ & 23 & 87 & 27 & $27 a$ & - & 82 & 28 & $28 a$ & 26 & 80 & & & & & & & & & 33 & - & 83 \\
\hline
\end{tabular}


Figure 2. Preparation of L-Cysteine $\beta$-thio-Glycosides.

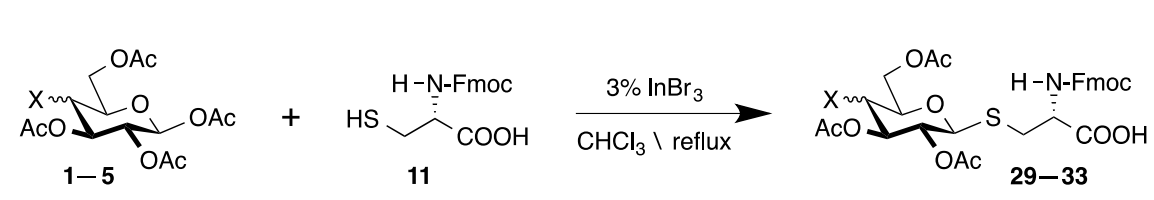
hydrolysis. Therefore, S-glycosylated amino acid building blocks compatible with common solid-phase peptide synthesis conditions are an attractive target. Fmoc-LCys(S-D-Gal)-OH has been previously reported by Kamerling et al, ${ }^{29}$ and Pieters et al, ${ }^{28}$ although their methodology required the use of excess $\mathrm{SnCl}_{4}$. We report similar yields using catalytic quantities of the minimally competent Lewis acid activator, and show the broad applicability of this approach through conjugation of five mono- and disaccharide glycosyl donors 29-33 to Fmoc-L-cysteine (Figure 2).

The importance of this study is twofold. First, only catalytic amounts of the Lewis acid promoter are required. Secondly, weakly electrophilic peracetates, generally regarded as synthetic intermediates rather than reactive donors, are suitable. This reduces the number of steps and greatly expands the application of this methodology. We have not observed the corresponding $\alpha$-thioglycosides in any of the reactions. 


\section{Experimental Section}

1.1 Materials and Instrumentation. ${ }^{1} \mathrm{H}$ and ${ }^{13} \mathrm{C}$ NMR spectra were recorded on a Bruker $400(400 \mathrm{MHz})$ spectrometer using $\mathrm{CDCl}_{3}$ and $\mathrm{D}_{3} \mathrm{COD}$ as solvents with TMS as the internal standard. All NMR spectra were analyzed and interpreted using MestReNova $^{\circledR}$ software. First-order chemical shifts and coupling constants were obtained from 1D spectra; proton and carbon resonances were assigned from COSY, HSQC and HMBC experiments. Mass spectrometry was achieved on a Thermo Finnigan LCQ Deca with positive and negative detection and a $1: 1 \mathrm{CH}_{3} \mathrm{OH}: \mathrm{H}_{2} \mathrm{O}$ solvent system. Optical rotation measurements were performed using a JASCO P-1010 polarimeter using the $\mathrm{Na} \mathrm{D}$ line $(589 \mathrm{~nm})$ in $\mathrm{MeOH}$ at room temperature, and specific rotation was determined using the JASCO software package. For flash chromatography Silia Flash P60 Silica Gel, 40-63 $\mu \mathrm{m}, 60 \AA$ (Silicycle, R1200308) was employed and a Biotage, Inc. Horizon HPFC system was utilized.

Preparative HPLC separations were performed on a Gilson HPLC using a Phenomenex C-18 reverse phase column (1000, $50 \times 250 \mathrm{~mm})$ with a flow rate of 25 $\mathrm{mL} / \mathrm{min}$ and detection at $280 \mathrm{~nm}$. Solvent systems A: $5 \%$ acetonitrile in $0.1 \%$ aqueous $\mathrm{F}_{3} \mathrm{CCOOH}$ and $\mathrm{B}$ : $80 \%$ acetonitrile in $0.1 \%$ aqueous $\mathrm{F}_{3} \mathrm{CCOOH}$ were used. A Dikma Technologies Inspire C-18 column $(4.6 \times 250 \mathrm{~mm})$ and a flow rate of $1.0 \mathrm{~mL} / \mathrm{min}$ was used for analytical HPLC separations. Thin layer chromatography (TLC) was performed on silica gel $60 \mathrm{~F} 254$ (Merck) with detection by UV light and charring with $\mathrm{H}_{2} \mathrm{SO}_{4}$. Solvents were dried and distilled according to literature procedures or purchased as anhydrous solvents from a manufacturer. All chemicals were purchased from Aldrich Chemical Company, Acros Chemicals, Chem-Impex International, or EMD Chemicals. 


\subsection{Alkyl- $\beta$-1-thio-D-glycopyranoside (12a-14a, 16a-28a). Peracetyl- $\beta$-D-}

glycopyranose (1-5, $10 \mathrm{mmol}), \mathrm{InBr}_{3}(106 \mathrm{mg}, 0.3 \mathrm{mmol}, 0.03 \mathrm{eq}$ ), and alkane-1-thiol (20 mmol, $2 \mathrm{eq}$ ) were suspended in $\mathrm{CHCl}_{3}(7 \mathrm{~mL})$. The reaction mixture was heated to reflux with magnetic stirring. The course of the reaction was followed by TLC (hexane:EtOAc 1:1). After 1 hour the peracetate starting material could no longer be detected by TLC. The reaction was cooled to RT, diluted with $\mathrm{CH}_{2} \mathrm{Cl}_{2}(20 \mathrm{~mL})$ and this solution was applied to a short column $(4 \mathrm{~cm} \times 12 \mathrm{~cm})$ of $\mathrm{SiO}_{2}$. The $\mathrm{HOAc}$ that was formed from the reaction and the excess alkane-1-thiol were eluted with hexanes. The desired thio-glycoside peracetate was eluted with hexanes:EtOAc 7:3. The fractions containing the desired material were combined, concentrated in vacuo, and the intermediate peracetates $(\mathbf{1 2 - 1 4}, \mathbf{1 6 - 2 8})$ were characterized by $1 \mathrm{D}, 2 \mathrm{D},{ }^{1} \mathrm{H}-\mathrm{NMR}$ and ${ }^{13} \mathrm{CNMR}$ in $\mathrm{CDCl}_{3}$ (in Tables).

Zemplén's method was used to deprotect the peracetates. ${ }^{18}$ The thioglycopyranoside peracetates were dissolved in anhydrous $\mathrm{CH}_{3} \mathrm{OH}(100 \mathrm{~mL})$ and the basicity was adjusted to $\mathrm{pH}=9$ by addition $\mathrm{NaOCH}_{3}$. After 16 hours the deacetylation was deemed complete by TLC. Dowex ${ }^{\circledR}$ resin $\left(\mathrm{H}^{+}\right.$form $)$was added until the $\mathrm{pH}$ was slightly acidic, the resin was filtered off, and the solution was concentrated, to provide 12a-14a, 16a-28a, which were characterized.

\subsection{1,10 Dithio- $\beta$-D-glucopyranosyl-decane (15a) Peracetyl- $\beta$-D-glucopyranose (25 $\mathrm{mmol}), \operatorname{lnBr}_{3}(3 \mathrm{mmol}, 0.03 \mathrm{eq})$, and decane-1,10-dithiol $(10 \mathrm{mmol}, 1 \mathrm{eq})$ were reacted using the previous method to provide $15 a$. M.p. $124-132^{\circ} \mathrm{C}$ (crystallization from $\mathrm{MeOH}$}


- $\mathrm{Et}_{2} \mathrm{O}$ ), $[\alpha]_{D}-36.5^{\circ}$ (c 0.13 , in $\mathrm{MeOH}$ ), MS (ESI HRMS) for $\mathrm{C}_{22} \mathrm{H}_{42} \mathrm{O}_{10} \mathrm{~S}_{2}(+\mathrm{Na})$ 553.21116, found 553.21195 [M+Na $]^{+}$.

1-Thio- $\beta$-D-glucopyranosyl-10-thiol-decane (16a) M.p. $95-97^{\circ} \mathrm{C}$ (crystallization from $\mathrm{MeOH}-\mathrm{Et}_{2} \mathrm{O}$ ), $[\alpha]_{\mathrm{D}}-17.8^{\circ}$ (c 0.20 , in $\left.\mathrm{MeOH}\right), \mathrm{MS}(\mathrm{ESI} H R M S)$ for $\mathrm{C}_{16} \mathrm{H}_{32} \mathrm{O}_{5} \mathrm{~S}_{2}(+\mathrm{Na})$ 391.15834, found $391.15875[\mathrm{M}+\mathrm{Na}]^{+}$.

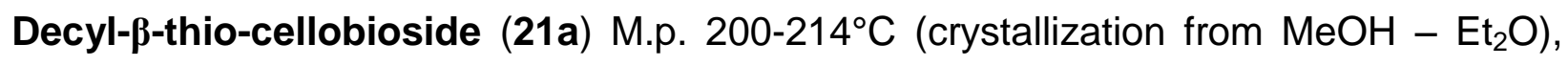
$[\alpha]_{D}-22.1^{\circ}$ (c 0.26, in $\left.\mathrm{MeOH}\right)$, MS (ESI HRMS) for : $\mathrm{C}_{22} \mathrm{H}_{42} \mathrm{O}_{10} \mathrm{~S}(+\mathrm{Na}) 521.23909$, found $521.24009[\mathrm{M}+\mathrm{Na}]^{+}$.

Decyl- $\beta$-thio-lactoside (27a) M.p. Decomp. $\sim 200^{\circ} \mathrm{C}$ (crystallization from $\mathrm{MeOH}-$ $\mathrm{Et}_{2} \mathrm{O}$ ), $[\alpha]_{D}-18.3^{\circ}$ (c 0.40 , in $\left.\mathrm{MeOH}\right)$, MS (ESI HRMS) for $\mathrm{C}_{22} \mathrm{H}_{42} \mathrm{O}_{10} \mathrm{~S}(+\mathrm{Na})$ 521.23909, found $521.24000[\mathrm{M}+\mathrm{Na}]^{+}$.

\section{4 $N^{\alpha}$-Fluoren-9-ylmethoxycarbonyl-S-(peracetyl- $\beta$-D-glyco-pyranosyl)-L-cysteine}

(29-33). Peracetyl- $\beta$-D-glycopyranose (0.6 mmol, 2eq), $\operatorname{lnBr}_{3}(21 \mathrm{mg}, 0.06 \mathrm{mmol}, 0.2$ eq), and $N^{\alpha}$-fluoren-9-ylmethoxycarbonyl-L-cysteine $(\mathbf{1 1}, 103 \mathrm{mg}, 0.3 \mathrm{mmol}, 1 \mathrm{eq})$ were suspended in $\mathrm{CHCl}_{3}(1.5 \mathrm{~mL})$. The reaction mixture was heated to reflux with magnetic stirring. The course of the reaction was followed by analytical HPLC. After 1 hour HPLC indicated $>80 \%$ conversion (see Table 1 ) of Fmoc-Cys $(\mathrm{SH})-\mathrm{OH}$ to the desired product. Additional reaction time yielded no further reaction progress that could be detected by HPLC. The reaction mixture was concentrated in vacuo, and the residue was purified by preparative HPLC on a $\mathrm{C}_{18}$ reverse phase column with $\mathrm{H}_{2} \mathrm{O}-\mathrm{CH}_{3} \mathrm{CN}$ gradient. The appropriate fractions were freeze-dried and the solid residue was characterized. 


\section{$N^{\alpha}$-Fluoren-9-ylmethoxycarbonyl-S-(peracetyl- $\beta$-D-cellobiosyl)-L-cysteine}

Phase transition point $104-105^{\circ} \mathrm{C}$ (lyophilization from $\mathrm{CH}_{3} \mathrm{CN} / \mathrm{H}_{2} \mathrm{O}$ ), $[\alpha]_{D}-20.7^{\circ}$ (c 0.15 , in $\mathrm{MeOH})$, MS (ESI HRMS) for $\mathrm{C}_{44} \mathrm{H}_{51} \mathrm{NO}_{21} \mathrm{~S}(+\mathrm{Na})$ 984.25665, found 984.25901 $[\mathrm{M}+\mathrm{Na}]^{+}$.

\section{$N^{\alpha}$-Fluoren-9-ylmethoxycarbonyl-S-(peracetyl- $\beta$-D-maltobiosyl)-L-cysteine}

Phase transition point $103-104{ }^{\circ} \mathrm{C}$ (lyophilization from $\mathrm{CH}_{3} \mathrm{CN} / \mathrm{H}_{2} \mathrm{O}$ ), $[\alpha]_{D}+21.5^{\circ}$ (c 0.14 , in $\mathrm{MeOH}$ ), MS (ESI HRMS) for $\mathrm{C}_{44} \mathrm{H}_{51} \mathrm{NO}_{21} \mathrm{~S}(+\mathrm{Na})$ 984.25665, found 984.25866 $[\mathrm{M}+\mathrm{Na}]^{+}$.

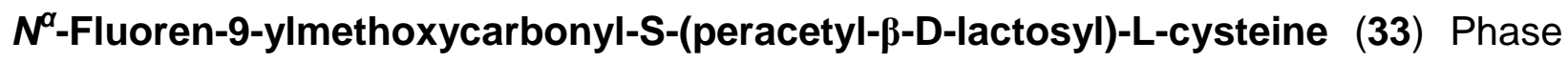
transition point $109-110{ }^{\circ} \mathrm{C}$ (lyophilization from $\mathrm{CH}_{3} \mathrm{CN} / \mathrm{H}_{2} \mathrm{O}$ ), $[\alpha]_{D}-20.6$ (c 0.17 , in $\mathrm{MeOH}), \mathrm{MS}(\mathrm{ESI} H R M S)$ for $\mathrm{C}_{44} \mathrm{H}_{51} \mathrm{NO}_{21} \mathrm{~S}(+\mathrm{Na}) 984.25665$, found $984.25817[\mathrm{M}+\mathrm{Na}]^{+}$.

Acknowledgements. We acknowledge support of this research through grants from the NSF/EPA Networks for Sustainable Molecular Design and Synthesis (NSMDS) program (CHE-1339597), the Michael J. Fox Foundation, and the Migraine Research Foundation. 


\section{References and Footnotes.}

1. Brown, D.A., London, E., J. Membr. Biol., 1998; 164:103-114.

2. Kitamoto, D., Morita, T., Fukuoka, T., Konishi, M.-a., Imura, T., Curr. Opin. Colloid In., 2009; 14:315-328.

3. Salim, M., Minamikawa, H., Sugimura, A., Hashim, R., Medchemcomm, 2014; 5:1602-1618.

4. Rodrigues, L.R., J. Colloid Interface Sci., 2015; 449:304-316.

5. Niraula, B.B., Chun, T.K., Othman, H., Misran, M., Colloid. Surface. A, 2004; 248:157-166.

6. Fischer, E., Delbrück, K., Ber. Dtsch. Chem. Ges., 1909; 42:1476-1482.

7. Macauley, M.S., Stubbs, K.A., Vocadlo, D.J., J. Am. Chem. Soc., 2005; 127:17202-17203.

8. Veeneman, G.H., Vanleeuwen, S.H., Vanboom, J.H., Tetrahedron Lett., 1990; 31:1331-1334.

9. Zhu, X., Schmidt, R.R., Angew. Chem. Int. Ed., 2009; 48:1900-1934.

10. Lloyd, D., Bennett, C.S., J. Org. Chem., 2014; 79:9826-9829.

11. Codée, J.D.C., Litjens, R., van den Bos, L.J., Overkleeft, H.S., van der Marel, G.A., Chem. Soc. Rev., 2005; 34:769-782.

12. Salvador, L.A., Elofsson, M., Kihlberg, J., Tetrahedron, 1995; 51:5643-5656.

13. Coss, C., Carrocci, T., Maier, R.M., Pemberton, J.E., Polt, R., Helv. Chim. Acta, 2012; 95:2652-2659.

14. Lefever, M.L., Szabo, L.Z., Anglin, B., Ferracane, M., Hogan, J., Cooney, L., Polt, R., Carbohydr. Res., 2012; 351:121-125.

15. Mattson, A.L., Michel, A.K., Cloninger, M.J., Carbohydr. Res., 2012;

347:142-146.

16. Keyari, C.M., Polt, R., J. Carbohydr. Chem., 2010; 29:181-206.

17. Saito, S., Tsuchiya, T., Chem. Pharm. Bull., 1985; 33:503-508.

18. Zemplén, G., Kunz, A., Ber. Dtsch. Chem. Ges., 1924; 57:1357-1359.

19. Galema, S.A., Engberts, J., vanDoren, H.A., Carbohydr. Res., 1997; 303:423434.

20. Van Doren, H.A., Van Der Geest, R., Kellogg, R.M., Wynberg, H., Carbohydr. Res., 1989; 194:71-78.

21. Yde, M., Debruyne, C.K., Carbohydr. Res., 1973; 30:205-206.

22. Dahlhoff, W.V., Radkowski, K., Riehl, K., Zugenmaier, P., Z. Naturforsch. B, 1995; 50:1079-1085.

23. Defaye, J., Gadelle, A., Pedersen, C., US Patent 5,118,804, 1991.

24. Mousty, C., Maurice, C., Mousset, G., Schöllhorn, B., Lefeuvre, M., Plusquellec, D., J. Colloid Interface Sci., 1996; 184:671-679. 
25. Kumpulainen, A.J., Persson, C.M., Eriksson, J.C., Langmuir, 2004; 20:1053410541.

26. Mori, M., Kasuya, M.C.Z., Mizuno, M., Hatanaka, K., Int. J. Carbohydr.

Chem., 2011; 8 pp.

27. Katayama, H., Asahina, Y., Hojo, H., J. Pept. Sci., 2011; 17:818-821.

28. Branderhorst, H.A., Liskamp, R.A.J., Pieters, R.J., Tetrahedron, 2007;

63:4290-4296.

29. Maljaars, C.E.P., Halkes, K.M., de Oude, W.L., van der Poel, S., Pijnenburg,

N.J.M., Kamerling, J.P., J. Carbohydr. Chem., 2005; 24:353-367.

30. Jones, E.M., Polt, R., Front. Chem. (Lausanne, Switz.), 2015; 3:40. 